\title{
The Spatiotemporal Dynamics of Low-abundance Bioactive Lipids in Arteries Undergoing Restenosis Observed and Identified at High Spatial Resolving Power with Multi-modal Mass Spectrometry Imaging
}

Gregory Fisher ${ }^{1}$, Yatao $\mathrm{Shi}^{2}$, Jillian Johnson ${ }^{2}$, Bowen Wang ${ }^{3}$, Bingming Chen ${ }^{2}$, Go Urabe ${ }^{3}$, Xudong Shi ${ }^{4}$, K. Craig Kent ${ }^{3}$, Lian-Wang $\mathrm{Guo}^{3}$ and Lingjun $\mathrm{Li}^{5}$

${ }^{1}$ Physical Electronics, Chanhassen, Minnesota, United States, ${ }^{2}$ School of Pharmacy, University of Wisconsin-Madison, Madison, Wisconsin, United States, ${ }^{3}$ Davis Heart and Lung Research Institute, The Ohio State University, Columbus, Ohio, United States, ${ }^{4}$ Department of Surgery, School of Medicine and Public Health, University of Wisconsin-Madison, Madison, Wisconsin, United States, ${ }^{5}$ Department of Chemistry, University of Wisconsin-Madison, Madison, Wisconsin, United States

The most common remedy for reduced or obstructed circulation is balloon angioplasty. While acutely effective, the treatment causes restenosis in a high fraction of patients via neointimal lesions. A significant barrier to unravelling the mechanisms of restenosis concerns the dynamic spatial profiling of bioactive lipids in the arterial wall [1]. Applying matrix-assisted laser desorption-ionization (MALDI) and time-offlight secondary ion mass spectrometry (TOF-SIMS) imaging, we conducted a first spatiotemporal lipidomic study of angioplasty-induced restenosis in a small animal model. The MS imaging data revealed that diacylglycerol (DG) and lyso-phosphatidylcholine (LPC) signals increased within the neointima layer of the 7- and 14-day injured specimens compared to the controls, while sphingomyelin (SM) signals decreased versus the controls.

Rat carotid arteries were collected 3-, 7- and 14-days after balloon angioplasty. Arterial injury was induced by three withdrawing passes, using a 2-F balloon catheter inflated to $2 \mathrm{~atm}$., from the proximal common carotid artery to the carotid bifurcation. Injured (left) and control (right) carotid specimens were harvested from the same animal and were used to generate $12 \mu \mathrm{m}$-thick sections for subsequent MS imaging analyses. MALDI was performed using an LTQ-Orbitrap XL spectrometer and a $75 \mu \mathrm{m}$ laser probe diameter at a power of $20 \mu \mathrm{J}$. TOF-SIMS tandem MS imaging was performed using a Parallel Imaging MS/MS spectrometer, with $\mathrm{keV}-\mathrm{CID}$, and a $\mathrm{Bi}_{3}{ }^{+}$nanoprobe operated at $<500 \mathrm{~nm}$ beam diameter (Figure 1).

Several DG, LPC and SM molecules were found to be diagnostic of the spatiotemporal lipid dynamics related to restenosis. Certain accurate mass molecular identifications had high uncertainties (i.e. $\Delta m / z \geq 4$ ppm); therefore, TOF-SIMS tandem MS imaging was used to identify these low abundance lipids owing to greater sensitivity [2], enhanced keV-CID molecular product ion generation [3], and high spatial resolving power. The accurate identification of low abundance molecules, e.g. $<20 \mathrm{ppm}$ abundance, was further enabled by single ion pulse counting and near-zero background wherein peaks of $\mathrm{S} / \mathrm{N} \geq 3$ could be used for product ion attributions (Figure 2). At areas about and away from the neointima lesions, making use of the high spatial resolving power, the keV-CID product ion spectra provided elucidation of isobaric molecules. At $m / z \approx 577.5$ three different lipid moieties were readily identified and appeared at different areas of the injured arteries; the ions were identified as $\mathrm{DG}(34: 1)\left[\mathrm{M}+\mathrm{H}-\mathrm{H}_{2} \mathrm{O}\right]^{+}, \mathrm{DG}(33: 3)[\mathrm{M}+\mathrm{H}]^{+}$and $\mathrm{DG}(31: 0)[\mathrm{M}+\mathrm{Na}]^{+}$. At $m / z \approx 575.5$ three different lipid moieties were identified but were localized together in the injured arteries; the ions were identified as DG(34:2) $\left[\mathrm{M}+\mathrm{H}-\mathrm{H}_{2} \mathrm{O}\right]^{+}, \mathrm{DG}(33: 4)[\mathrm{M}+\mathrm{H}]^{+}$and 
$\mathrm{DG}(31: 1)[\mathrm{M}+\mathrm{Na}]^{+}$. It is noteworthy that these ions differ by only one unsaturation; nevertheless, the spatial distributions are distinct.

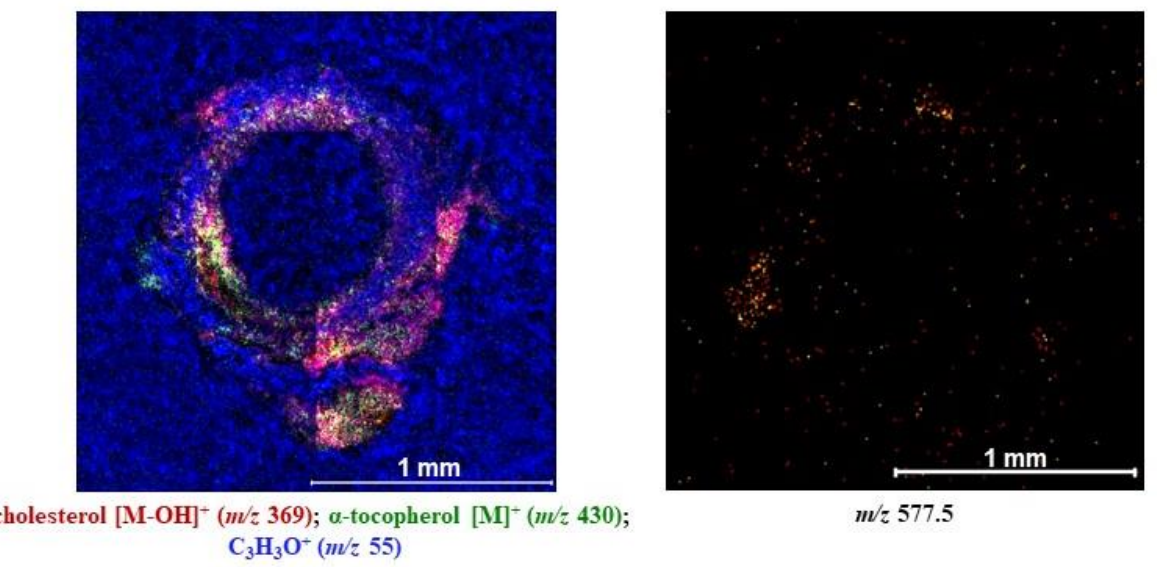

Figure 1. TOF-SIMS tandem MS images of an injured artery section at 7-days post-angioplasy. (LEFT) False color overlay image of the injured artery section of cholesterol $[\mathrm{M}-\mathrm{OH}]^{+}(\mathrm{m} / \mathrm{z} 369$, red), vitamin E $\left[\mathrm{M}^{+}\left(\mathrm{m} / \mathrm{z}, 430\right.\right.$, green) and a fragment ion $\mathrm{C}_{3} \mathrm{H}_{3} \mathrm{O}^{+}(\mathrm{m} / \mathrm{z}, 55$, blue) of the embedding material. (RIGHT) Thermal-scaled image of the molecular ions appearing at $\mathrm{m} / z .577 .5$ and which are shown to consist of three different DG structures. Certain molecules appearing at $m / z 577.5 \pm 0.25$ have an abundance $£ 20$ ppm.

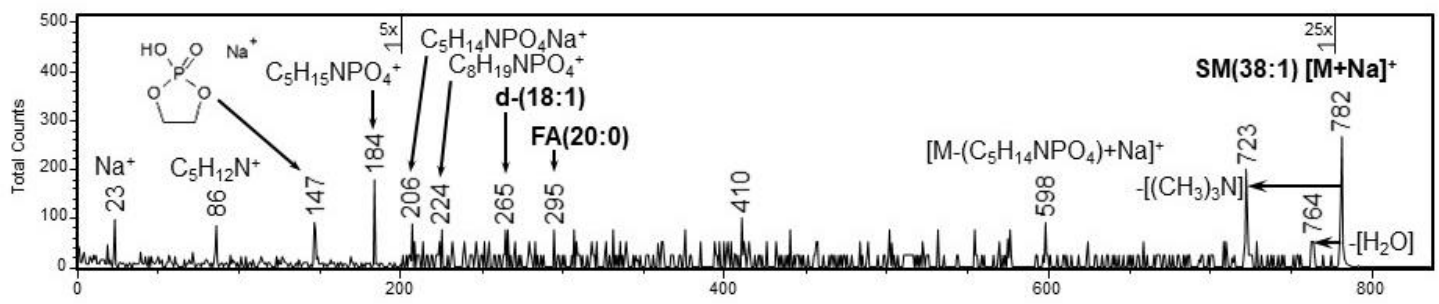

Figure 2. TOF-SIMS keV-CID product ion spectrum of the precursor ion at $\mathrm{m} / \mathrm{z} 781.62$ identified as $\mathrm{SM}(38: 1)[\mathrm{M}+\mathrm{Na}]^{+}$due to the diagnostic product ions at $\mathrm{m} / z$ 147, 184, 265, 295, 598 and 723 corresponding to the sodiated $O, O^{\prime}$-dimethylenephosphoric acid ion, the phosphocholine headgroup ion, the acyl ion of FA(18:1), the acyl ion of FA(20:0), the product ion produced by neutral loss (183 Da) of the phosphocholine, and the product ion produced by neutral loss $(59 \mathrm{Da})$ of trimethylamine, respectively. The ion fluence was $1.41 \times 10^{11} \mathrm{Bi}^{+} / \mathrm{cm}^{2}$.

\section{References}

[1] Y. Shi, et al., J. Proteome Res. 18 (2019) 1669.

[2] L. MacAleese, et al, J. Proteom. 74 (2011) 993.

[3] (a) G.L. Fisher, et al, Anal. Chem. 88 (2016) 6433. (b) G.L. Fisher, et al, Microscop. Microanal. 23 (2017) 843. (c) N. Ogrinc Potočnik, et al, Anal. Chem. 89 (2017) 8223. 